\title{
Do genetic differences in growth thermal reaction norms maintain genetic variation in timing of diapause induction?
}

\author{
Erlend I. F. Fossen $^{1,2}$ (D) | Joost A. M. Raeymaekers ${ }^{1,3}$ (i) | Sigurd Einum ${ }^{1}$
}

${ }^{1}$ Department of Biology, Centre for Biodiversity Dynamics, NTNU, Norwegian University of Science and Technology, Trondheim, Norway

${ }^{2}$ Animal Ecology, Department of Ecology and Genetics, Uppsala University, Uppsala, Sweden

${ }^{3}$ Faculty of Biosciences and Aquaculture, Nord University, Bodø, Norway

\section{Correspondence}

Erlend I. F. Fossen, Animal Ecology, Department of Ecology and Genetics, Uppsala University, Uppsala 752 36, Sweden.

Email: erlend.fossen@ebc.uu.se

Funding information

Norwegian University of Science and Technology; Research Council of Norway, Grant/Award Number: 223257/F50; Research Council of Norway, FRIPRO programme, Grant/Award Number: 230482

\section{Abstract}

1. An optimal timing for diapause induction through the sexual production of dormant propagules is expected in organisms with temporary populations. Yet, empirical studies often find high within-population genetic variation in the sexual production of such propagules, suggesting that this is a common feature of such organisms.

2. Here, we hypothesize that genetic variation in the propensity to produce dormant propagules, $P_{d}$, is maintained by a genotype-by-environment interaction in clonal reproductive rates, where fast-growing genotypes within an environment should delay diapause relative to slow-growing genotypes. From this, we derive two predictions. First, if reaction norms of clonal reproduction cross between two environments, the genetic correlation of $P_{d}$ between these environments should be negative. Second, the correlation between plasticity values of clonal reproduction and $P_{d}$ should be negative.

3. We tested these predictions by quantifying ephippia production in genotypes of a population of the facultative sexual cladoceran Daphnia magna at two temperatures. The population biomass at the onset of ephippia production was used as a measure of $P_{d}$, whereas juvenile somatic growth rate was used as a proxy for clonal reproductive rate. Plasticity for both measurements was derived from thermal reaction norms.

4. Our results did not support either prediction, as neither the genetic correlation of $P_{d}$ between environments, nor the correlation between plasticity values of growth and $P_{d}$ were found to be significant.

5. Our results suggest that genetic variation in the timing of diapause is not maintained by genetic differences in thermal clonal reproduction reaction norms. We propose as an alternative hypothesis that if there is variation across years in how the environment deteriorates over a season, fluctuating selection may favor genotypes with different $P_{d}$ between years.

\section{KEYWORDS}

diapause, genotype-by-environment interaction, reaction norm, resting eggs, temperature 


\section{1 | INTRODUCTION}

The timing of reproduction is a crucial life history trait (Stearns, 2000). This is particularly true for many organisms living in temperate environments, where conditions for successful growth and reproduction are limited to certain time horizons (Gotthard, 2001). These time horizons can be interspersed by periods preventing survival (e.g. winter or dry seasons), in which case production of dormant propagules is required. Examples of these dormant propagules are seeds in plants and diapause eggs in animals like killifish (Murphy \& Collier, 1997), cladocerans (Frey, 1960), rotifers (García-Roger et al., 2017), copepods (Holm et al., 2018) and some insects (e.g. silkworms, Tirelli, 1946). For these organisms, the timing of the switch from somatic growth and/or asexual reproduction to the production of dormant propagules has strong fitness consequences, and there is an optimal timing of sexual reproduction (Cohen, 1976). Delaying onset of sexual reproduction allows for more time to grow and higher reproductive output per reproductive event, but comes at the cost of reducing the number of reproductive events or risking reproductive failure (Furness et al., 2015; Weis et al., 2014). Organisms with facultative parthenogenesis, which can switch between asexual and sexual reproduction (e.g. cladocerans [Taylor \& Gabriel, 1993; Gerber et al., 2018], rotifers [Serra et al., 2008; García-Roger et al., 2017] and aphids [Simon et al., 2002]), represent a special case. For these, the optimal timing involves an allocation trade-off between investing in biomass through repeated events of clonal reproduction versus ensuring production of dormant propagules before the growing season ends.

Genetic variation in the timing of flowering and subsequent seed production is commonly observed in plants (e.g. Bourion et al., 2002; Franks et al., 2007; Hara \& Ohsawa, 2013). Similarly, for facultative parthenogenetic animals which typically switch from asexual to sexual reproduction when environmental conditions deteriorate (but see Serra et al., 2004 for alternative explanations where the timing of sexual reproduction matches favorable environmental conditions and optimal male-female encounter rates), there is genetic variation in the propensity to produce dormant propagules $\left(P_{d}\right)$ in a given environment, suggesting that genotypes vary in their environmental cue thresholds (e.g. Carmona et al., 2009; Deng, 1996; Gilbert, 2002; Roulin et al., 2015; Yampolsky, 1992). In a seasonal environment, such variation in cue thresholds should translate into variation in timing of the switch. Thus, given the predicted optimal switch time within a population, explaining the maintenance of such genetic variation in $P_{d}$ is a challenge. Whereas diversified bet-hedging may produce phenotypic variation within genotypes as an adaptation to unpredictable environmental variation (Botero et al., 2015; Tufto, 2015), it should not maintain variation among genotypes. Environmental variation may however contribute to maintaining genetic variance non-adaptively through imposing temporally fluctuating selection and hence preventing fixation of optimal alleles (Sasaki \& Ellner, 1997). Thus, one possible explanation for the genetic variation in $P_{d}$ is that the way by which the environment deteriorates throughout the season varies across years, which in turn may cause the optimal $P_{d}$ to vary.

An alternative hypothesis for maintaining genetic variation in $P_{d}$ is that different genotypes are adapted to different environmental conditions that occur during different parts of the season, and thus show different responses to environmental gradients in their ability to accumulate biomass (i.e. genotype-by-environment [G $\times E$ ] interactions in somatic growth or clonal reproduction, e.g. Carvalho, 1987; Fossen et al., 2018; Kingsolver et al., 2004). If this $\mathrm{G} \times \mathrm{E}$ interaction is strong, it can generate an "ecological crossover" where different genotypes are superior in different environments (Ellner \& Hairston, 1994; Gillespie \& Turelli, 1989; Higginson \& Reader, 2009; Turelli \& Barton, 2004). This should lead to corresponding differences in $P_{d}$ across environments, and different optimal timing of making the switch from asexual to sexual reproduction in an environment that declines in quality towards the end of the season (Figure 1). For example, a genotype with low clonal reproduction rate in cold environments would benefit from having a higher $P_{d}$ when exposed to a low temperature, compared to a genotype that is able to maintain a high clonal reproduction at that low temperature. Similar patterns have been observed for other genetically correlated traits, where these show corresponding $\mathrm{G} \times \mathrm{E}$ interactions (e.g. Prati \& Schmid, 2000; Stinchcombe et al., 2004; Mills et al., 2007). For example, Prati and Schmid (2000) showed a genetic tradeoff between flowering and rooting (i.e. between sexual reproduction and clonal growth), where flowering showed a $G \times E$ interaction that corresponded with a $G \times E$ interaction in rooting. Such a trade-off might also apply in the case of clonal versus sexual reproduction in facultative parthenogenetic animals, because the same female reproductive organs are used for both types of reproduction. Thus, according to this hypothesis, two predictions can be derived regarding the patterns of genetic variance in $P_{d}$ (Figure 2). First, if there is ecological crossover in clonal reproductive rates (Figure 2a), genotypes have similar reproduction rates in an intermediate environment. From an optimality perspective this also means that they should have similar values of $P_{d}$ in the same intermediate environment (Figure 2b). Moving in one direction away from this intermediate environment, genotypes that increase clonal reproduction relative to other genotypes should simultaneously reduce their relative values of $P_{d}$. This in turn should lead to a negative genetic correlation between $P_{d}$ across environments (Figure 2c). Second, there should be a negative genetic correlation between the plasticity value of $P_{d}$ and the plasticity value of clonal reproduction (Figure $2 d$ ). The plasticity value is here defined as the slope of the trait reaction norm. The second prediction is based on the assumption that for a given genotype, the direction of change in $P_{d}$ across environments should be opposite of the direction of change in clonal reproduction, while the relative magnitude in change should be similar (e.g. a genotype with a steeper positive clonal reproduction slope compared to other genotypes is predicted to have a steeper negative $P_{d}$ slope than other genotypes). Considering that clonal reproduction and $P_{d}$ are under a trade-off to maximize final dormant propagule production, having steeper slopes 

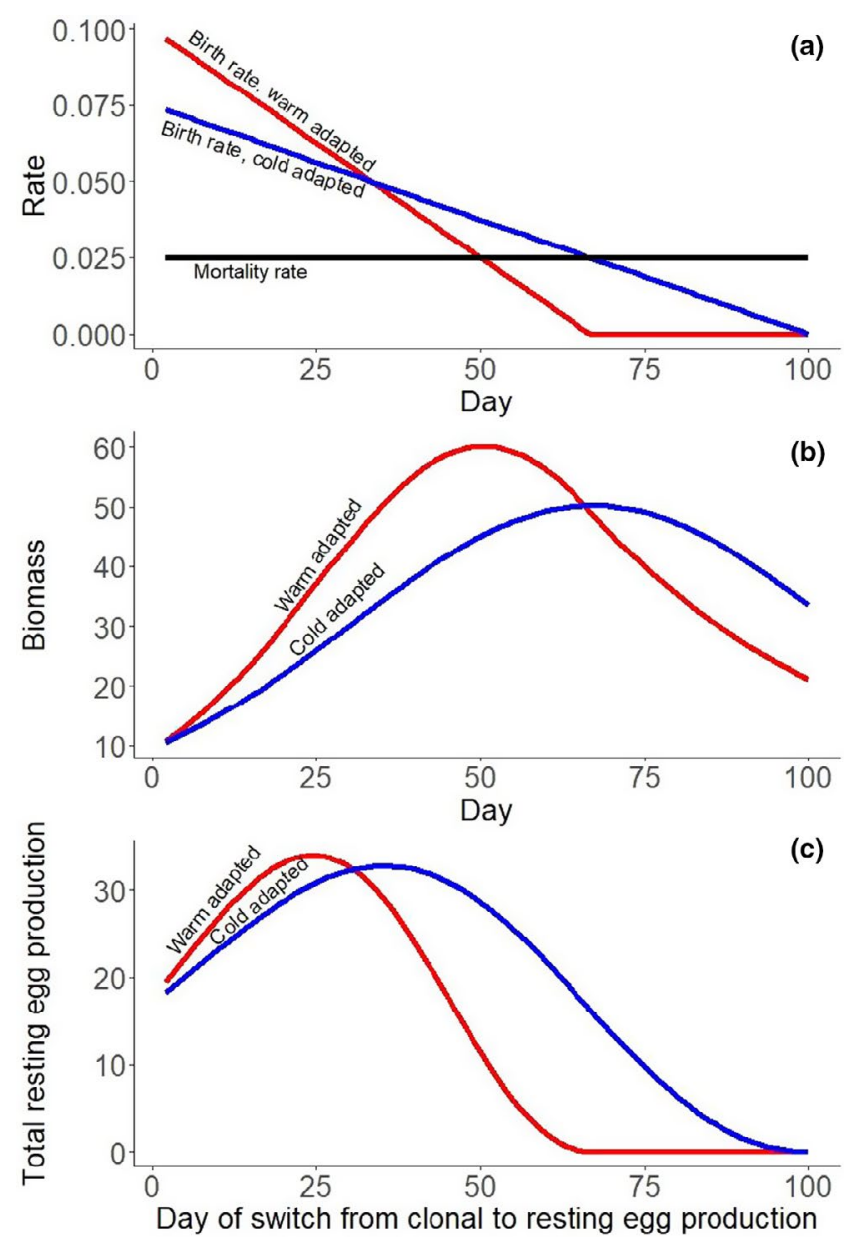

FIGURE 1 A modelled example of the temporal dynamics towards the end of the season (i.e. under declining temperatures) for an organism that switches from asexual (clonal) to sexual reproduction (production of dormant propagules). The population consists of two genotypes that (a) differ in their response of birth rates to the declining temperature due to a strong $\mathrm{G} \times \mathrm{E}$ interaction (i.e. ecological crossover). (b) In the absence of switching, the two genotypes reach their peak biomass at different days. (c) The total (cumulative) production of dormant propagules by the end of the season as a function of the day that the genotype makes the switch. For these latter calculations, no asexual production occurs after the switch is made, and hence population biomass declines according to the mortality rate. The following models and parameter values are used (a) Birth rate cold adapted $=0.075-0.00075 \times$ Day, Birth rate warm adapted $=0.1-0.0015 \times$ Day, Mortality rate $=0.025$, (b) Biomass ${ }_{i, t+1}=$ Biomass $_{i, t} \times\left(1+\right.$ Birth rate $_{i, t}-$ Mortality rate), where $i$ indicates genotype, Biomass ${ }_{0}=10$, (c) Dormant propagule production $_{i, t}=$ Biomass $_{i, t} \times$ Birth rate $_{i, t}$ if $t \geq$ switch day, $^{2}$ otherwise 0

implies investing a lot in one or the other trait, depending on the environment.

To increase the understanding of what maintains genetic variation in the timing of diapause induction we tested these predictions by studying a population of the facultative sexual cladoceran Daphnia magna, a species that is known to harbor pronounced genetic variance in dormant propagule production (Roulin et al., 2015;
Yampolsky, 1992). The population used originates from a pond at its northern distribution limit, where production of dormant propagules during autumn is expected to be crucial for overwintering fitness. Using a set of ten genetically distinct genotypes, we ran population growth experiments at different temperatures and quantified production of dormant propagules through ephippia counts. Population density is one important cue that Daphnia use to switch to ephippia production (Gyllström \& Hansson, 2004; Kleiven et al., 1992). The population density, measured as population biomass, required to trigger this switch was therefore used as an inverse measure of $P_{d}$ at the different temperatures. Furthermore, thermal reaction norms of juvenile somatic growth rates for the same clones (Fossen et al., 2018) were used to test for a genetic correlation between environmental cue thresholds and clonal reproduction. For Daphnia, there is a close covariance between juvenile somatic growth rate and clonal reproduction rate across temperatures and food rations (e.g. $r^{2}=0.95$ in Rinke \& Petzoldt, 2003; $r^{2}=0.55-0.99$ in Lampert \& Trubetskova, 1996). Thus, thermal reaction norms of somatic growth rate represent a good proxy for reaction norms of clonal reproduction.

\section{2 | METHODS}

\section{1 | Study animals and husbandry of stock cultures}

Ephippia of Daphnia magna Straus, 1,820, which contain up to two sexually produced dormant propagules, were collected in November 2014 from the surface sediment of a shallow pond at Værøy Island (Sandtjønna, 1.0 ha, $67.687^{\circ} \mathrm{N} 12.672^{\circ} \mathrm{E}$ ), northern Norway. This pond freezes over during winter and its mean daily water temperatures during summer fluctuates between $10-20^{\circ} \mathrm{C}$ (Figure 2 in Fossen et al., 2018). Ten genotypes, hatched from separate ephippia in December 2014, were cultured separately under common garden conditions for 2-3 years (>50 asexual generations). These genotypes, hereafter referred to as clones, vary genetically in thermal plasticity of life-history traits (Fossen et al., 2018). Daphnids were kept in $250 \mathrm{ml}$ jars containing a modified ADaM medium (Klüttgen et al., $1994, \mathrm{SeO}_{2}$ concentration reduced by $50 \%$, sea-salt increased to $1.23 \mathrm{~g} / \mathrm{L}$ ) at $17^{\circ} \mathrm{C}$ with a $16 \mathrm{~L}: 8 \mathrm{D}$ photoperiod, and the medium was changed weekly. Cultures contained five female adults per $250 \mathrm{ml} \mathrm{jar}$ and were fed three times a week with Shellfish Diet 1,800 (Reed Mariculture Inc.) at a final algae concentration of $4 \times 10^{5}$ cells $\mathrm{m} / \mathrm{L}$.

\section{2 | Experimental design}

Daphnia use several environmental cues to switch from clonal growth and reproduction to production of dormant propagules, including population density/food abundance, photoperiod, and temperature (e.g. Deng, 1996; Gyllström \& Hansson, 2004; Kleiven et al., 1992; Slusarczyk \& Rybicka, 2011). Of these, we kept photoperiod constant (16L:8D, representing autumn conditions in the 
population's native environment), while experimentally manipulating temperature. The experiment (January - June 2017, where experimental populations were started across 23 dates) consisted of two temperature treatments $\left(12\right.$ and $22^{\circ} \mathrm{C}$ ). These temperatures were chosen based on an exploratory study (Appendix S1) that showed that they are $5^{\circ} \mathrm{C}$ below and above the temperature that triggered a change in mean ephippia production (Figure S1), and should enable testing of our predictions.

A total of 163 replicate populations were studied, with 7-9 replicates per clone per temperature (Table S1). Clones were kept in replicated lines at their experimental temperatures for two or more asexual generations prior to experiments to ensure acclimation. A single female juvenile ( $<24 \mathrm{hr}$ old from the second clutch) was used to initiate each replicate experimental population. All temperature treatments (acclimation and experiment) were created by placing $250 \mathrm{ml}$ jars in climate cabinets (IPP 260plus; Memmert). Throughout the acclimation and experimental period, animals were fed temperature-specific food concentrations every second day (concentrations: $12^{\circ} \mathrm{C}, 2.00 \times 10^{5}$ cells m/L; $22^{\circ} \mathrm{C}, 3.24 \times 10^{5}$ cells $\mathrm{m} / \mathrm{L}$ ) and the medium was refreshed by sieving every 8 days at $12^{\circ} \mathrm{C}$ and every 4 days at $22^{\circ} \mathrm{C}$. This food regime represents ad libitum concentrations when the population size is low (Appendix S2), and ensures comparable starting conditions across temperatures. The same food regime was used in Fossen et al. (2018) to obtain somatic growth rates, thus making these two studies directly comparable. An alternative food regime with equal food concentrations and medium refreshments across temperatures was considered an option, but would result in suboptimal starting conditions for growth at one of the temperatures, less comparable water quality across temperatures, and results that are less comparable to the somatic growth rate estimates from Fossen et al. (2018). Considering that the predictions are tested based on the pattern of genetic variance within temperatures (see Statistical Analyses section), different food regimes across temperatures should not affect the conclusions.

Each experimental population was checked daily until both the second clutch was born and until the shedding of the first ephippium. During this phase, we obtained the timing and number of offspring of the two first clutches, and the timing of the onset of ephippia production. After this phase, and at the onset of ephippia production, we recorded a six seconds video of each population at every second medium change (camera: Panasonic DMC-TZ25, Full $\mathrm{HD}, 1,920 \times 1,080$ pixels). For the video, all animals of a population were transferred to a transparent glass baking dish, which was put on top of a light table to obtain a high contrast between animals and the background. These videos were later used to estimate population sizes and body size distributions over time using the Rpackage trackdem v. 0.3.1 (Bruijning et al., 2018). Videos made by us $(N=153)$ under the same conditions as described above were used by Bruijning et al. (2018) to evaluate the accuracy of trackdem. This analysis revealed highly accurate and unbiased estimates of the population size (Figure 3 in Bruijning et al., 2018). Trackdem uses automated particle tracking to keep track of the number of moving particles (here: individual daphnids) of potentially different size, in addition to outputting the size of the particles (number of pixels). By taking videos of animals that were not from the experiment, but raised under the same conditions, we obtained an equation for the relationship between dry mass (DM, $\mathrm{mg}$ ) of individual animals and their particle size $(D M=-0.00635+0.00100 \times$ particle size, $n=21$, $\left.R^{2}=0.953\right)$. The dry mass of these animals was first estimated from their carapax length $(\mathrm{CL}, \mathrm{mm})$ using a known length - dry mass relationship for our population ( $\mathrm{DM}=0.00535 \mathrm{CL}^{2.72}$, Yashchenko et al., 2016). Thus, using videos from the experiment and estimated individual particle sizes, we could estimate total population size, the number of adults (animals $>0.075 \mathrm{mg}$ dry mass, based on size at maturity in Fossen et al., 2018) and the total biomass at any time a video was recorded. During each medium change, all shed ephippia were counted and removed (to prevent dormant propagules from hatching during the experiment). The experiments were terminated on day 128 at $12^{\circ} \mathrm{C}$ and on day 56 at $22^{\circ} \mathrm{C}$, based on the results from the exploratory study (Appendix $\mathrm{S} 1$ ). This corresponds approximately to twice the time it took populations to reach peak population density (where ephippia production also peaked) in the exploratory study.

\section{3 | Trait measurements}

\subsection{1 | Ephippia production}

Ephippia production in D. magna involves production of dormant propagules that require fertilization by males to be viable. Thus, for a given clone, producing few ephippia could potentially be compensated by predominately producing males (that fertilize receptive females from other clones), resulting in a negative correlation between production of males and production of ephippia. In the presence of such alternative reproductive tactics, a high production of males could be an alternative way to achieve genetic contribution to the diapause stage at the population level. Our design that kept clones separately could then potentially introduce noise when using ephippia production as a measure of $P_{d}$. However, a previous study showed no such correlation among clones within populations of D. magna, and rather a positive correlation among populations (Roulin et al., 2015). Furthermore, we tested for this possibility in our own population by calculating the proportion of males of each population at the end of the exploratory study (Appendix S1). Although the proportion of males (mean $[S D]=0.025$ [0.037]) differed significantly among clones (logistic GLM with clone as fixed effect, $p<0.001$ ), there were no significant genetic correlations between the proportion of males and total ephippia production at any temperature (range: [-0.24, 0.23], $p>0.5$ for all temperatures). This is a common finding for Daphnia (e.g. Lampert et al., 2012; Roulin et al., 2015; Yampolsky, 1992). Finally, the observation that an approximately 50:50 sex ratio is attained in populations under strong stimuli by production of alternating male and female clutches within females (Hobæk \& Larsson, 1990), suggests that the presence of 
alternative reproductive tactics is unlikely. Thus, we only consider ephippia production in further analyses. parable across temperatures, we calculated ephippia produced per generation for each population as follows: ephippia per generation $=\frac{\text { total number of ephippia }}{\text { duration/generation time }}$, where duration is the duration of the time series, and the average time to first clutch of each temperature was used as a proxy for temperature-specific generation time. The total number of ephippia is given in Figure S2a.

\subsubsection{Estimating $P_{d}$}

Population density and food availability are known cues used by Daphnia to switch from growth to ephippia production (Gyllström \& Hansson, 2004; Kleiven et al., 1992). Thus, population biomass, which should better represent food availability than population density, should also be a valid cue for the switch. Thus, population biomass at the onset of ephippia production (Figure S2b) was used as an inverse measure of $P_{d}$ (i.e. a high population biomass required to trigger the switch to ephippia production represents a low $P_{d}$ ). To make this comparable across temperatures (which differed in food rations) we divided this measure with the temperature specific food rations. By making this normalization we assume that food availability, rather than biomass per se, is the cue that triggers ephippia production. While this may not completely control for differences in food ration,
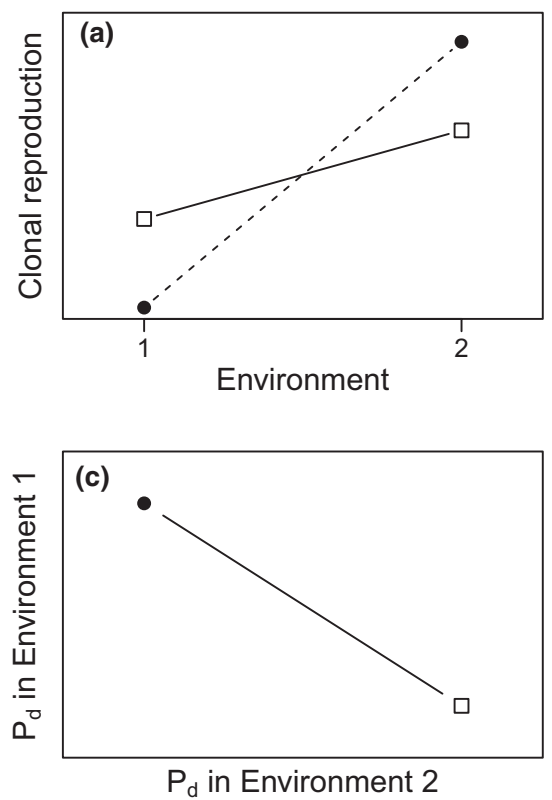

To get a measurement of ephippia production that is com-

we note that all populations became food limited when reaching high population density and produced ephippia. Consequently, $P_{d}$ could be estimated for each population. Furthermore, the pattern of genetic variance (e.g. ranking of clones) within temperatures is not affected by this normalization of $P_{d^{*}}$. Although we did not have biomass measurements in the exploratory study (Appendix S1), a correlated measure (population density at the onset of ephippia production, Figure S3a,b) was positively genetically correlated across exploratory versus main experiments (Figure S3c,d), indicating that this measurement is repeatable across experiments.

\subsection{3 | Clonal reproduction}

We used clone-specific estimates of thermal reaction norms for juvenile somatic growth rate, obtained from Fossen et al. (2018), as a proxy for thermal reaction norms of clonal reproductive rates (see Introduction). An ecological crossover in somatic growth rate was found by Fossen et al. (2018), where reaction norms of somatic growth rate crossed at $14^{\circ} \mathrm{C}$ (Figure S4). Clone-specific slope estimates of growth performance across temperatures were used to test our second prediction (as in Figure 2a,d).

\subsection{Statistical analyses}

All analyses were conducted in R v.3.3.1 (R Core Team, 2014).
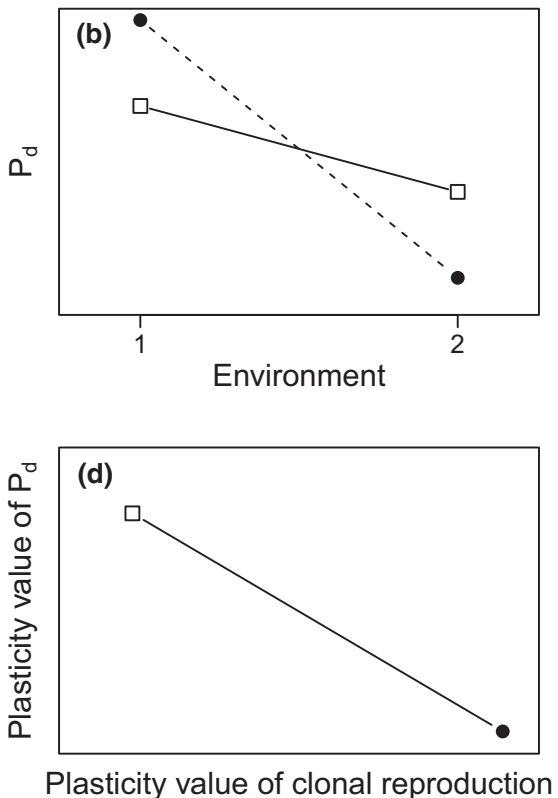

FIGURE 2 Predictions following the hypothesis that variation in reaction norms of clonal reproduction can maintain genetic variation in the propensity to produce dormant propagules $\left(P_{d}\right)$. If there is a strong $\mathrm{G} \times \mathrm{E}$ interaction with ecological crossover in clonal reproduction (a, two genotypes represented by different symbols), reaction norms for $P_{d}$ are expected to also show ecological crossover (b). Consequently, a negative genetic correlation is predicted between $P_{d}$ in the two environments (c). Furthermore, we predict a negative genetic correlation between the plasticity values of these traits, where the plasticity value is defined as the slope of the trait reaction norm (d). This follows from the hypothesis that genotypes that experience a stronger decrease in clonal reproduction when going from environment 2 to environment 1 should also have a higher increase in $P_{d}$ 


\subsection{1 | Total ephippia production - temperature response, genetic variance and correlation with $P_{d}$}

We used linear mixed-effect models with the package Ime4 (v. 1.1-7, Bates et al., 2015) to test for genetic variance in total ephippia production across the two temperatures. Ephippia per generation was the response variable, and temperature was included as a categorical predictor variable. Clone and start date (of experimental populations) were used as random effects. An interaction between clone effect and temperature effect was assumed in the full model (i.e., amongclone variance could vary with temperature), as well as a clone effect on the intercept. Start date was assumed to only affect the overall intercept. Akaike information criterion corrected for small sample sizes (AICc) were used in model selection and to access significance of effects (factors that only showed up in models $>2 \Delta \mathrm{AICc}$ were considered non-significant). Full models with different random effect were first compared using restricted maximum likelihood (REML), and then the best random effect structure was used when comparing models with different fixed effects using maximum likelihood. Lastly, parameter estimates were obtained from the best model fitted with REML. Pseudo- $R^{2}$ values were calculated as the squared correlation coefficient between fitted values from the model and observed values.

To test if having a smaller $P_{d}$ resulted in lower total ephippia production, we calculated genetic correlations between the two traits within each of the two temperatures, using temperature specific clonal means.

2.4.2 | Prediction 1: G $\times$ E interactions and a genetic correlation between $P_{d}$ across environments

To estimate genetic variance and test for $\mathrm{G} \times \mathrm{E}$ interactions in $P_{d}$, we used mixed effect models with biomass per food abundance at the onset of ephippia production as the response variable. The same models and model procedures were applied as for ephippia production (see above). We then proceeded with testing for a negative genetic correlation between $P_{d}$ across the two temperatures (as in Figure 2c). We note that even if the estimated biomass per food abundance does not completely control for differences in food ration across temperatures, the mean-standardized genetic variance and order of clones within temperatures do not change due to the normalization of $P_{d}$, and neither does the genetic correlation across temperatures (data not shown).

\subsection{3 | Prediction 2: Genetic correlation between the plasticity values of $P_{d}$ and of clonal reproduction}

Our second prediction was that there should be a negative genetic correlation between the plasticity value of $P_{d}$ and the plasticity value of clonal reproduction (Figure $2 \mathrm{~d}$ ). We quantified the plasticity value of $P_{d}$ as the negative of the slope (i.e. slope multiplied with -1) between biomass per food abundance at the onset of ephippia production and temperature, such that a clone with a large positive slope-value requires a relatively stronger population-density cue at low temperatures than at high temperatures. As for prediction 1 , since genetic variance within temperatures is not affected by the normalization of $P_{d}$, neither is the genetic correlation between plasticity values affected (even though the reaction norm slopes change).

\subsection{4 | Evolutionary potential}

To get an estimate of the population's evolutionary potential that is comparable across traits, populations and species, the broad sense evolvability (clonal variance/mean ${ }^{2}$ ) was calculated within temperatures (Hansen et al., 2003, 2011; Houle, 1992). This was done for both ephippia production and $P_{d}$. Evolvability is a measurement of the expected percentage change in a trait per generation under a unit strength of selection, and is (in contrast to heritability) independent from environmental variance (Hansen et al., 2011).

\section{3 | RESULTS}

\section{1 | Total ephippia production - temperature response, genetic variance and correlation with $P_{d}$}

Ephippia production was on average about eight times larger at 12 than $22^{\circ} \mathrm{C}$ (Figure 3). We found a significant $\mathrm{G} \times \mathrm{E}$ interaction in ephippia production (Table S2), with higher broad sense evolvability at $12^{\circ} \mathrm{C}$ (clonal variance $\left[\mathrm{V}_{\text {clone }}\right]=51.34$ [ephippia per generation] ${ }^{2}$, evolvability $=14.27 \%)$ than at $22^{\circ} \mathrm{C}\left(\mathrm{V}_{\text {clone }}=0.08\right.$, evolvability $=2.74 \%$ ). Variance due to start date was 12.67 (ephippia per generation) ${ }^{2}$, the residual variance was 19.49 , and the model pseudo- $R^{2}$ was 0.86 . Furthermore, the variation in ephippia production among clones was highly repeatable across exploratory versus main experiments at $12^{\circ} \mathrm{C}$ (Figure S1b), but not at $22^{\circ} \mathrm{C}$ (Figure S1c).

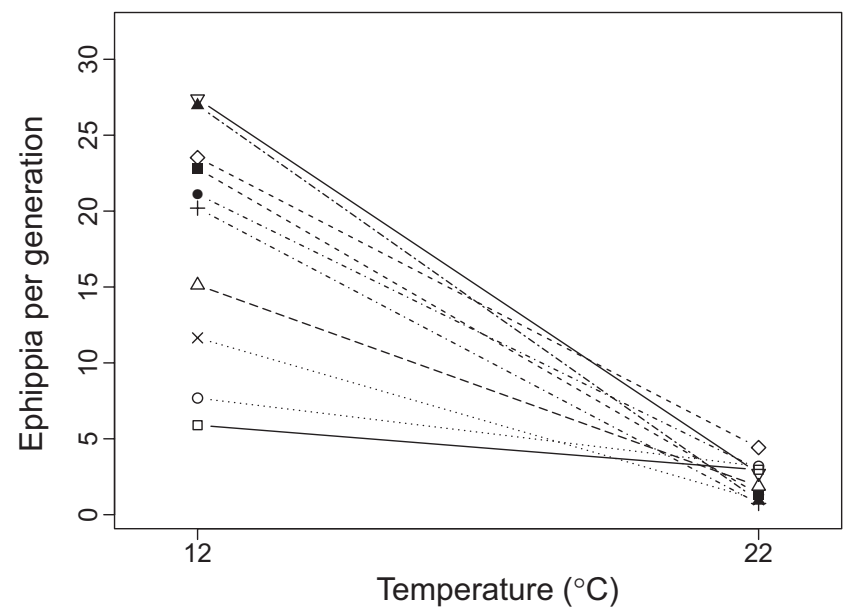

FIGURE 3 Ephippia production per generation across temperature for 10 clones of a population of Daphnia magna. For procedures on calculation see Methods. Each point is the mean of a clone, represented by different symbols 
Ephippia production at $12^{\circ} \mathrm{C}$ increased for all populations after about 60 days (Figure S5), shortly after peak population density (Figure 4). At $12^{\circ} \mathrm{C}$ but not at $22^{\circ} \mathrm{C}, P_{d}$ was positively genetically correlated with ephippia production (Figure $\mathrm{S} 6$ ).

\subsection{Prediction 1: $\mathrm{G} \times \mathrm{E}$ interactions and a genetic correlation between $P_{d}$ across environments}

$P_{d}$ showed a significant $G \times E$ interaction with temperature, with some clones having a particularly low propensity to produce dormant propagules at low temperatures (Figure 5a, Table S2). The overall broad sense evolvability was higher at $12^{\circ} \mathrm{C}$ (clonal variance $\left[\mathrm{V}_{\text {clone }}\right]=1664.4\left[\mathrm{ng} /\left[\text { cell ml } \mathrm{ml}^{-1} \mathrm{day}^{-1}\right]\right]^{2}$, evolvability $=35.45 \%)$ than at $22^{\circ} \mathrm{C}\left(\mathrm{V}_{\text {clone }}=2.2\right.$, evolvability $\left.=0.08 \%\right)$. The model pseudo- $R^{2}$ was 0.75 , variance due to start date was $117.0\left(\mathrm{ng} /\left[\mathrm{cell} \mathrm{ml^{-1 }} \mathrm{day}^{-1}\right)^{2}\right.$ and the residual variance was 414.5. However, in contrast to the prediction of a negative genetic correlation between $P_{d}$ at 12 versus $22^{\circ} \mathrm{C}$, the genetic correlation of the trait across temperature was not significant (Figure $5 \mathrm{~b} ; r=0.41$, $\mathrm{t}_{8}=1.26, p=0.243$ ).

\section{3 | Prediction 2: Genetic correlation between the plasticity values of $P_{d}$ and the plasticity of clonal reproduction}

While we expected the plasticity value of $P_{d}$ to decrease with increasing plasticity value of somatic growth rate, the relationship between the traits was not significant (Figure 6; $r=0.29, \mathrm{t}_{8}=0.855$, $p=0.417)$

\section{4 | DISCUSSION}

To test if genetic differences in thermal clonal reproduction reaction norms can maintain genetic variation in the timing of diapause induction, we ran population growth experiments using clones from
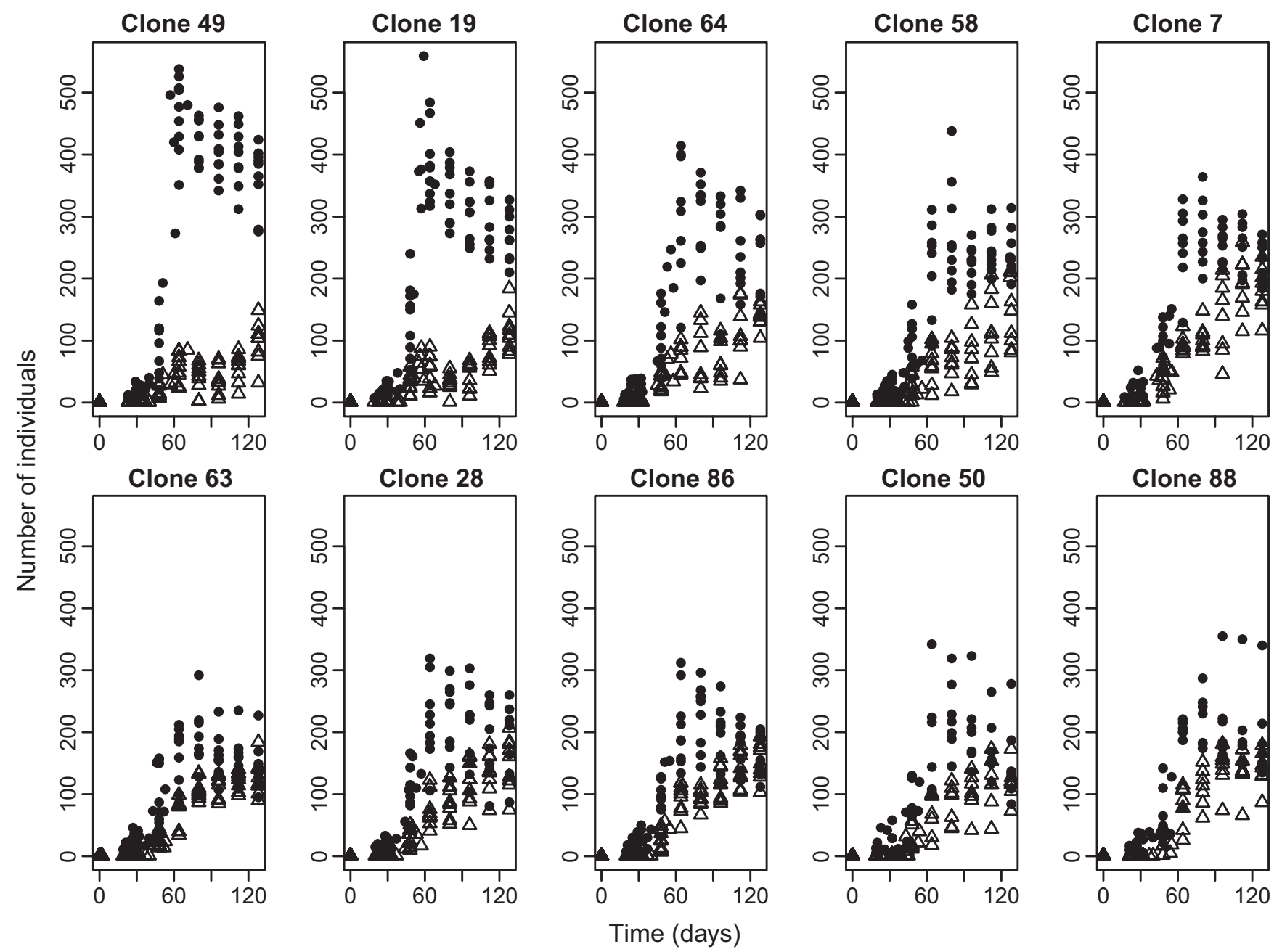

FIGURE 4 Time series showing an association between ephippia production (panels sorted by increasing ephippia production from top left to bottom right) and age structure in 10 clones of Daphnia manga at $12^{\circ} \mathrm{C}$. Filled circles represent the total population size, triangles the number of adults. There are multiple observations per time point, because 7-9 replicates were used per clone 

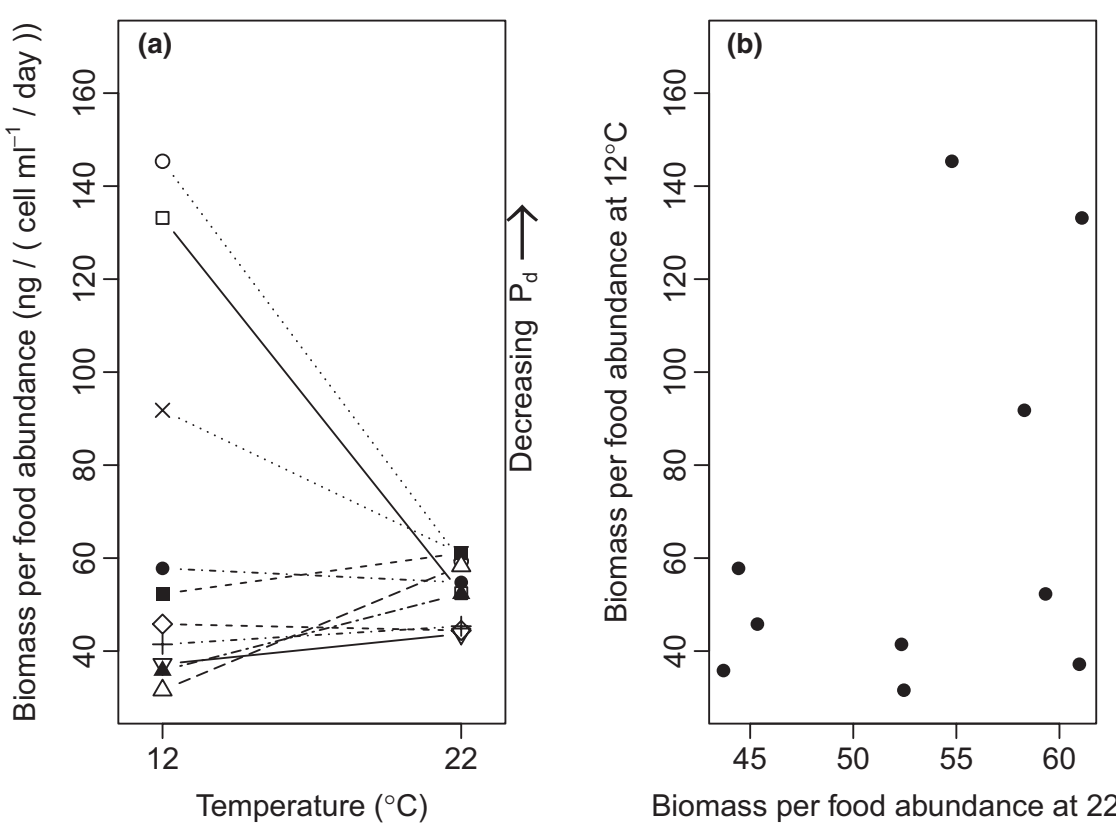

FIGURE 5 Propensity to produce dormant propagules $\left(P_{d}\right)$ for 10 clones of a population of Daphnia magna. $P_{d}$ was measured as population biomass per food abundance at the onset of ephippia production, where a low biomass represents a high $P_{d}$. There is a significant $G \times E$ interaction (a), but no significant genetic correlation across temperatures (b, $r=0.41, \mathrm{t}_{8}=1.26, p=0.243$ )

Biomass per food abundance at $22^{\circ} \mathrm{C}$

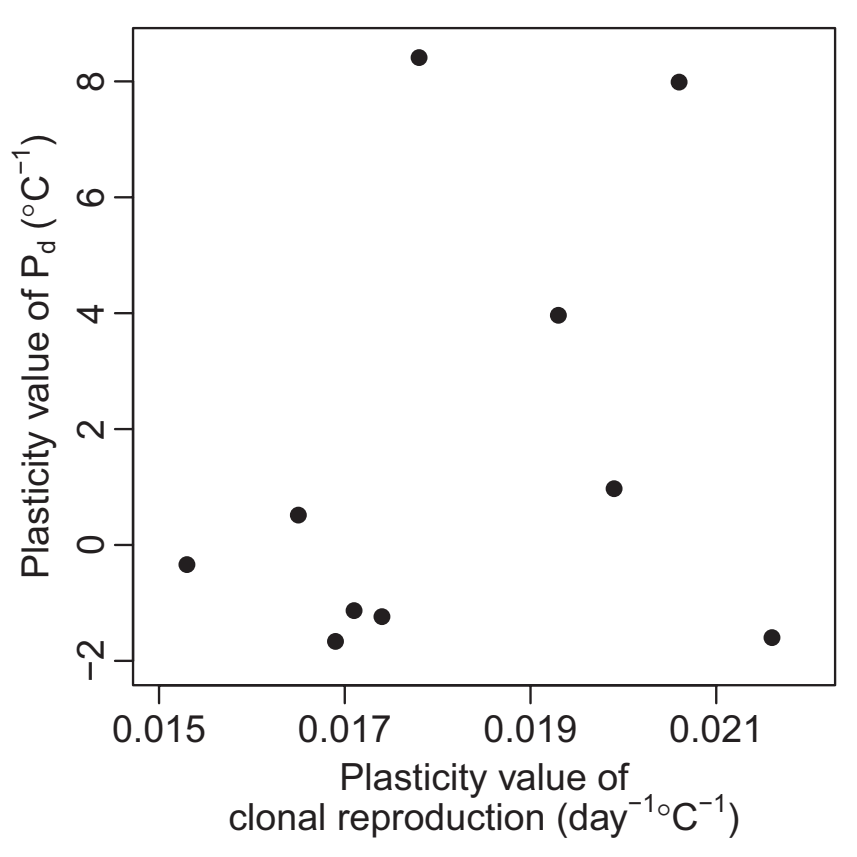

FIGURE 6 No significant genetic correlation $(r=0.29$, $\mathrm{t}_{8}=0.855, p=0.417$ ) between thermal plasticity in the propensity to produce dormant propagules $\left(P_{d}\right)$ and a clonal reproduction trait (somatic growth rate). Each point shows the plasticity value of the traits, representing the mean change in the trait per ${ }^{\circ} \mathrm{C}$ for a given clone of Daphnia magna. The plasticity value of clonal reproduction was measured as the slope of the traits reaction norm, whereas the plasticity value of $P_{d}$ was measured as the negative of the slope between biomass per food abundance at the onset of ephippia production and temperature

a single population of Daphnia magna. We quantified relationships between ephippia production, the propensity to produce dormant propagules $\left(P_{d}\right)$, and a growth performance trait that is closely correlated with clonal reproductive rates in Daphnia (juvenile somatic growth rate) across two temperatures. Our results showed that there was high genetic variance in $P_{d}$, and $P_{d}$ was positively genetically correlated with total ephippia production at $12^{\circ} \mathrm{C}$. Whereas both traits showed higher evolvability $\left(>10 \%\right.$ at $\left.12^{\circ} \mathrm{C}\right)$ than the mean of that found in wild populations (about $1 \%$ for life history traits, Hansen et al., 2011), particularly at $12^{\circ} \mathrm{C}$, the estimates were well within the range of previously reported values $(<0.001 \%$ to $>100 \%)$. The observed differences among clones at $12^{\circ} \mathrm{C}$ were also highly consistent with results from a separate exploratory study. Having found such large differences among clones in $P_{d}$, we tested a hypothesis derived from the observation that genotypes within the same population can be adapted to different seasons (e.g. Carvalho, 1987; Grosberg, 1988), i.e. that genotypes should match genotype-specific environmental variation in $P_{d}$ with corresponding variation in their asexual production. We tested two predictions derived from this hypothesis. First, we predicted a negative genetic correlation between $P_{d}$ across a temperature range that encompass ecological crossover in asexual production. Second, we predicted a negative genetic correlation between the thermal plasticity value of clonal production and the thermal plasticity value of $P_{d}$. Our results, showing no significant correlations, did not support either of the predictions. This lack of statistical support is unlikely to be due to low statistical power since the signs of the correlations were opposite of what was predicted from the hypothesis. This indicates that genetic differences in growth thermal reaction norms do not maintain genetic variation in $P_{d}$.

Although we failed to provide support for our predictions, certain conditions may have prevented this. For instance, if the temperature treatments were too similar, this could prevent detection of a negative correlation between $P_{d}$ across temperatures (prediction 1). The low genetic variation in $P_{d}$ at $22^{\circ} \mathrm{C}$ suggest that this may have been the case and that measuring $P_{d}$ at higher temperatures could have shown a clearer ecological crossover. However, if there is a trade-off between clonal reproduction (and hence juvenile somatic growth) and ephippia production, growth and $P_{d}$ should show similar crossover 
points (see Introduction). Considering that thermal reaction norms for somatic growth rate cross at a temperature intermediate to our two experimental temperatures, $P_{d}$ is also predicted to cross at an intermediate temperature. Moreover, ephippia production, which showed large differences at the tested temperatures and a similar pattern across temperature as $P_{d}$, also showed similar levels of phenotypic and genetic variation at $22^{\circ} \mathrm{C}$ compared to $>22^{\circ} \mathrm{C}$, suggesting that the temperature treatments should be sufficiently different to detect any genetic correlation between $P_{d}$ across temperatures. Alternatively, if juvenile somatic growth rate was a poor measure of asexual population growth, a genetic correlation between the plasticity value of somatic growth and the plasticity value of $P_{d}$ would not be predicted. This does however seem unlikely considering that this measure of somatic growth rate has previously been strongly linked to the intrinsic rate of population increase in our study species (Lampert \& Trubetskova, 1996). Overall, for both predictions to fail simultaneously, two independent conditions would need to occur (too similar test-environments and using an inappropriate trait as a measure of clonal reproduction), which seems unlikely.

An alternative explanation for the maintenance of high genetic variation in $P_{d}$ is that there is strong fluctuating selection (across years) on this trait. This can occur if there is variation across years in to what extent temperature regimes deviate from a smooth seasonal trend of spring increase and subsequent fall decrease. In years with high levels of stochastic deviation from such a trend, short periods of low temperatures are likely to be followed by subsequent increases that allows for continued high asexual growth under low population densities. This will lead to lost opportunities for genotypes that have a high $P_{d}$, and hence rapidly switch to ephippia production in the absence of strong competition (i.e. at low population density). Selection should then favor clones that have a low $P_{d}$ and require stronger population density cues to commence ephippia production during such years. At the other extreme, for years with no stochasticity a decline in temperature is always followed by a further decline. In this situation, selection should favor clones that do not wait and see whether temperature conditions improve following a decline, but that have a high $P_{d}$ and rapidly engage in ephippia production when exposed to a certain decline in temperature. Such maintenance of genetic variation due to temporally fluctuating selection is similar to other cases where the optimum trait value fluctuates over time (e.g. timing of diapause in copepods, Hairston \& Dillon, 1990; morph color and pattern in stick insects, Nosil et al., 2018). These strategies may also explain the low genetic variance at $22^{\circ} \mathrm{C}$ compared to at $12^{\circ} \mathrm{C}$. If temperature conditions are favorable, all clones should invest in growth until their growth rate is constrained to a certain level due to high competition, resulting in all clones requiring a similar and high population density threshold to initiate ephippia production. It is important to note that this potential explanation relates to predictability throughout the season within single years. Thus, it is different from variability and predictability in mean conditions (i.e. season length) across years. At this latter scale, an unpredictable environment should select for conservative bet hedging strategies (Starrfelt $\&$ Kokko, 2012), where genotypes with high $P_{d}$ can be considered to be generalists and should be favored by selection due to their relative stable fitness across years, and hence high geometric mean fitness. Assessing the role of conservative bet hedging in evolution of $P_{d}$ is beyond the scope of the current study, as this would require a comparison among populations with different degrees of amongyear predictability in the environment (where higher $P_{d}$ is expected in populations with more unpredictable environments).

Declines in temperature have been suggested as a cue for the onset of winter and for inducing diapause in freshwater zooplankton in temperate areas (Gyllström \& Hansson, 2004; Slusarczyk \& Rybicka, 2011). Our finding of ephippia production being much higher at 12 than at $22^{\circ} \mathrm{C}$ supports this. Yet, the median biomass per food abundance needed to induce ephippia production was similar at the two temperatures. This may seem surprising, but can be explained by the fact that ectotherms at higher temperatures have higher metabolic rates and therefore also higher food demands (also shown for our Daphnia clones, Fossen et al., 2019). This explanation is supported by the observation that populations were able to maintain a much higher mean biomass per food abundance after the first ephippium had been produced at $12^{\circ} \mathrm{C}$ (mean $\pm S E=162.0 \pm 3.3 \mathrm{ng} /$ [cell $\mathrm{ml}^{-1} \mathrm{day}^{-1}$ ]) than that at $22^{\circ} \mathrm{C}($ mean $\pm S E=65.6 \pm 1.5)$.

In this study, we tested if a strong $G \times E$ interaction in clonal reproduction can maintain genetic variation in the propensity to produce dormant propagules. Despite finding high genetic variance in $P_{d}$, and using a clonal reproduction trait (somatic growth rate) that showed a strong $G \times E$ interaction, we found no support for this hypothesis. $P_{d}$ showed no genetic correlation across temperature (prediction 1), and there was no genetic correlation between the plasticity value of clonal reproduction and the plasticity value of $P_{d}$ (prediction 2). We propose that genetic variance in $P_{d}$, and hence the timing of a switch from clonal to sexual reproduction in natural populations, can be maintained by fluctuating selection, favoring genotypes that respond either quickly or more slowly in producing dormant propagules when experiencing declines in temperature, depending on how stochastically the temperature changes throughout the season. It can be expected that the stochasticity of seasonal temperature change will vary from year to year, particularly for temperate populations, and even more so with increases in extreme climatic events because of climate change. Consequently, variation in the relative success of these strategies across years may explain genetic variation in timing of diapause induction for a wide range of annual organisms. Moreover, this variation may be beneficial for populations to survive increases in extreme climatic events.

\section{ACKNOWLEDGMENTS}

Financial support was provided by the Research Council of Norway, FRIPRO programme, project 'Eco-evolutionary dynamics of thermal reaction norms' (Project 230482), and partly by the Research Council of Norway through its Centres of Excellence funding scheme, project number 223257/F50 and the Norwegian University of Science and Technology (NTNU). We thank V. Yashchenko, H.-K. Lakka, M. Jeannot, V. Parry, A. Vold, and others for help with data collection and culture maintenance. 


\section{DATA AVAILABILITY STATEMENT}

Data is archived in Dryad Digital Repository (https://doi.org/ 10.5061/dryad.0k6djhb19). The code for the model of Figure 1 is available upon request.

\section{ORCID}

Erlend I. F. Fossen (iD https://orcid.org/0000-0002-5687-2743

Joost A. M. Raeymaekers (iD https://orcid.

org/0000-0003-2732-7495

Sigurd Einum (iD https://orcid.org/0000-0002-3788-7800

\section{REFERENCES}

Bates, D., Mächler, M., Bolker, B., \& Walker, S. (2015). Fitting linear mixed-effects models using Ime4. Journal of Statistical Software, 67, 48.

Botero, C. A., Weissing, F. J., Wright, J., \& Rubenstein, D. R. (2015). Evolutionary tipping points in the capacity to adapt to environmental change. Proceedings of the National Academy of Sciences of the United States of America, 112, 184-189.

Bourion, V., Fouilloux, G., Le Signor, C., \& Lejeune-Hénaut, I. (2002). Genetic studies of selection criteria for productive and stable peas. Euphytica, 127, 261-273.

Bruijning, M., Visser, M. D., Hallmann, C. A., \& Jongejans, E. (2018). TRACKDEM: Automated particle tracking to obtain population counts and size distributions from videos in R. Methods in Ecology \& Evolution, 9, 965-973.

Carmona, M. J., Dimas-Flores, E., García-Roger, E. M., \& Serra, M. (2009). Selection of low investment in sex in a cyclically parthenogenetic rotifer. Journal of Evolutionary Biology, 22, 1975-1983.

Carvalho, G. R. (1987). The clonal ecology of Daphnia magna (Crustacea: Cladocera): II. Thermal differentiation among seasonal clones. Journal of Animal Ecology, 56, 469-478.

Cohen, D. (1976). The optimal timing of reproduction. The American Naturalist, 110, 801-807.

Deng, H.-W. (1996). Environmental and genetic control of sexual reproduction in Daphnia. Heredity, 76, 449-458.

Ellner, S., \& Hairston, N. G. (1994). Role of overlapping generations in maintaining genetic variation in a fluctuating environment. The American Naturalist, 143, 403-417.

Fossen, E. I. F., Pélabon, C., \& Einum, S. (2018). An empirical test for a zone of canalization in thermal reaction norms. Journal of Evolutionary Biology, 31, 936-943.

Fossen, E. I. F., Pélabon, C., \& Einum, S. (2019). Genetic and environmental effects on the scaling of metabolic rate with body size. Journal of Experimental Biology, 222, jeb193243. https://doi.org/10.1242/ jeb.193243

Franks, S. J., Sim, S., \& Weis, A. E. (2007). Rapid evolution of flowering time by an annual plant in response to a climate fluctuation. Proceedings of the National Academy of Sciences of the United States of America, 104, 1278-1282.

Frey, D. G. (1960). The ecological significance of cladoceran remains in lake sediments. Ecology, 41, 684-699.

Furness, A. I., Lee, K., \& Reznick, D. N. (2015). Adaptation in a variable environment: Phenotypic plasticity and bet-hedging during egg diapause and hatching in an annual killifish. Evolution, 69, 1461-1475.

García-Roger, E. M., Carmona, M. J., \& Serra, M. (2017). Modes, mechanisms and evidence of bet hedging in rotifer diapause traits. Hydrobiologia, 796, 223-233.

Gerber, N., Kokko, H., Ebert, D., \& Booksmythe, I. (2018). Daphnia invest in sexual reproduction when its relative costs are reduced. Proceedings of the Royal Society B: Biological Sciences, 285, 20172176.
Gilbert, J. J. (2002). Endogenous regulation of environmentally induced sexuality in a rotifer: A multigenerational parental effect induced by fertilisation. Freshwater Biology, 47(9), 1633-1641. https://doi. org/10.1046/j.1365-2427.2002.00900.x

Gillespie, J. H., \& Turelli, M. (1989). Genotype-environment interactions and the maintenance of polygenic variation. Genetics, 121, 129-138.

Gotthard, K. (2001). Growth strategies of ectothermic animals in temperate environments. In D. Atkinson, \& M. Thorndyke (Eds.), Environment and Animal Development (pp. 287-304): BIOS Scientific Publishers.

Grosberg, R. K. (1988). Life-history variation within a population of the colonial ascidian Botryllus schlosseri. I. The genetic and environmental control of seasonal variation. Evolution, 42, 900-920.

Gyllström, M., \& Hansson, L.-A. (2004). Dormancy in freshwater zooplankton: Induction, termination and the importance of benthicpelagic coupling. Aquatic Sciences, 66, 274-295.

Hairston, N. G., \& Dillon, T. A. (1990). Fluctuating selection and response in a population of freshwater copepods. Evolution, 44, 1796-1805.

Hansen, T. F., Pelabon, C., Armbruster, W. S., \& Carlson, M. L. (2003). Evolvability and genetic constraint in Dalechampia blossoms: Components of variance and measures of evolvability. Journal of Evolutionary Biology, 16, 754-766.

Hansen, T. F., Pelabon, C., \& Houle, D. (2011). Heritability is not evolvability. Journal of Evolutionary Biology, 38, 258-277.

Hara, T., \& Ohsawa, R. (2013). Accurate evaluation of photoperiodic sensitivity and genetic diversity in common buckwheat under a controlled environment. Plant Production Science, 16, 247-254.

Higginson, A. D., \& Reader, T. (2009). Environmental heterogeneity, genotype-by-environment interactions and the reliability of sexual traits as indicators of mate quality. Proceedings of the Royal Society B: Biological Sciences, 276, 1153-1159.

Hobæk, A., \& Larsson, P. (1990). Sex determination in Daphnia magna. Ecology, 76, 2255-2268.

Holm, M. W., Kiørboe, T., Brun, P., Licandro, P., Almeda, R., \& Hansen, B. W. (2018). Resting eggs in free living marine and estuarine copepods. Journal of Plankton Research, 40, 2-15.

Houle, D. (1992). Comparing evolvability and variability of quantitative traits. Genetics, 130, 195-204.

Kingsolver, J. G., Ragland, G. J., \& Shlichta, J. G. (2004). Quantitative genetics of continuous reaction norms: Thermal sensitivity of caterpillar growth rates. Evolution, 58, 1521-1529.

Kleiven, O. T., Larsson, P., \& Hobaek, A. (1992). Sexual reproduction in Daphnia magna requires 3 stimuli. Oikos, 65, 197-206.

Klüttgen, B., Dülmer, U., Engels, M., \& Ratte, H. T. (1994). ADaM, an artificial freshwater for the culture of zooplankton. Water Research, 28, 743-746.

Lampert, W., Lampert, K. P., \& Larsson, P. (2012). Coexisting overwintering strategies in Daphnia pulex: Clonal differences in sexual reproduction. Fundamental and Applied Limnology / Archiv Fur Hydrobiologie, 179, 281-291.

Lampert, W., \& Trubetskova, I. (1996). Juvenile growth rate as a measure of fitness in Daphnia. Functional Ecology, 10, 631-635.

Mills, S. C., Alatalo, R. V., Koskela, E., Mappes, J., Mappes, T., \& Oksanen, T. A. (2007). Signal reliability compromised by genotype-byenvironment interaction and potential mechanisms for its preservation. Evolution, 61, 1748-1757.

Murphy, W. J., \& Collier, G. E. (1997). A molecular phylogeny for aplocheiloid fishes (Atherinomorpha, Cyprinodontiformes): The role of vicariance and the origins of annualism. Molecular Biology and Evolution, 14, 790-799.

Nosil, P., Villoutreix, R., de Carvalho, C. F., Farkas, T. E., Soria-Carrasco, V., Feder, J. L., Crespi, B. J., \& Gompert, Z. (2018). Natural selection and the predictability of evolution in Timema stick insects. Science, $359,765-770$. 
Prati, D., \& Schmid, B. (2000). Genetic differentiation of life-history traits within populations of the clonal plant Ranunculus reptans. Oikos, 90, 442-456.

R Core Team. (2014). R: A language and environment for statistical computing: R Foundation for Statistical Computing.

Rinke, K., \& Petzoldt, T. (2003). Modelling the effects of temperature and food on individual growth and reproduction of Daphnia and their consequences on the population level. Limnologica, 33, 293-304. https://doi.org/10.1016/S0075-9511(03)80024-5

Roulin, A. C., Mariadassou, M., Hall, M. D., Walser, J. C., Haag, C., \& Ebert, D. (2015). High genetic variation in resting-stage production in a metapopulation: Is there evidence for local adaptation? Evolution, 69, 2747-2756. https://doi.org/10.1111/evo.12770

Sasaki, A., \& Ellner, S. (1997). Quantitative genetic variance maintained by fluctuating selection with overlapping generations: Variance components and covariances. Evolution, 51, 682-696.

Serra, M., Aparici, E., \& Carmona, M. J. (2008). When to be sexual: Sex allocation theory and population density-dependent induction of sex in cyclical parthenogens. Journal of Plankton Research, 30, 1207-1214.

Serra, M., Snell, T. W., \& King, C. E. (2004). The timing of sex in cyclically parthenogenetic rotifers. In A. Moya, \& E. Font (Eds.), Evolution: From molecules to ecosystems (pp. 135-146): Oxford University Press.

Simon, J.-C., Rispe, C., \& Sunnucks, P. (2002). Ecology and evolution of sex in aphids. Trends in Ecology and Evolution, 17, 34-39.

Slusarczyk, M., \& Rybicka, B. (2011). Role of temperature in diapause response to fish kairomones in crustacean Daphnia. Journal of Insect Physiology, 57, 676-680.

Starrfelt, J., \& Kokko, H. (2012). Bet-hedging-a triple trade-off between means, variances and correlations. Biological Reviews, 87, 742-755.

Stearns, S. C. (2000). Life history evolution: Successes, limitations, and prospects. Naturwissenschaften, 87, 476-486.

Stinchcombe, J. R., Dorn, L. A., \& Schmitt, J. (2004). Flowering time plasticity in Arabidopsis thaliana: A reanalysis of Westerman \& Lawrence (1970). Journal of Evolutionary Biology, 17, 197-207.
Taylor, B., \& Gabriel, W. (1993). Optimal adult growth of Daphnia in a seasonal environment. Functional Ecology, 7, 513-521.

Tirelli, M. (1946). Phenomena that occur in the centrifuged eggs of the silkworm Bombyx mori L. Physiological Zoology, 19, 346-354.

Tufto, J. (2015). Genetic evolution, plasticity, and bet-hedging as adaptive responses to temporally autocorrelated fluctuating selection: A quantitative genetic model. Evolution, 69, 2034-2049.

Turelli, M., \& Barton, N. (2004). Polygenic variation maintained by balancing selection: Pleiotropy, sex-dependent allelic effects and GxE interactions. Genetics, 166, 1053-1079.

Weis, A. E., Wadgymar, S. M., Sekor, M., \& Franks, S. J. (2014). The shape of selection: Using alternative fitness functions to test predictions for selection on flowering time. Evolutionary Ecology, 28, 885-904.

Yampolsky, L. Y. (1992). Genetic variation in the sexual reproduction rate within a population of a cyclic parthenogen, Daphnia magna. Evolution, 46, 833-837.

Yashchenko, V., Fossen, E. I., Kielland, Ø. N., \& Einum, S. (2016). Negative relationships between population density and metabolic rates are not general. Journal of Animal Ecology, 85, 1070-1077.

\section{SUPPORTING INFORMATION}

Additional supporting information may be found in the online version of the article at the publisher's website.

How to cite this article: Fossen, E. I. F., Raeymaekers, J. A. M., \& Einum, S. (2021). Do genetic differences in growth thermal reaction norms maintain genetic variation in timing of diapause induction? Freshw Biol, 66, 2185-2195.

https://doi.org/10.1111/fwb.13825 